\title{
Desing of a Chattering-Free Sliding Mode Control System for Robust Position Control of a Quadrotor
}

\author{
Aydin Can \\ Engineering Department \\ Lancaster University \\ Lancaster, UK \\ a.j.can@lancaster.ac.uk
}

\author{
Harry Efstathiades \\ Engineering Department \\ Lancaster University \\ Lancaster, UK \\ h.efstathiades@lancaster.ac.uk
}

\author{
Allahyar Montazeri \\ Engineering Department \\ Lancaster University \\ Lancaster, UK \\ a.montazeri@lancaster.ac.uk
}

\begin{abstract}
In this paper, the major components of an unmanned aerial vehicle (UAV), including the flight control system, mechanical design, as well as the embedded electronic systems are reported. The ultimate aim is to use the developed platform in nuclear environments for decommissioning applications. Due to the hazards and uncertainties present in these nuclear environments, a more basic control system may be unable to reliably control the UAV. Therefore, the main focus of this study is on the development of a robust multi-channel control system, based on sliding mode control (SMC), for nested position and attitude control of a quadrotor $\mathrm{UAV}$ in $3 \mathrm{D}$ space. The robustness of the control systems is evaluated under parameter disturbance in the form of payload uncertainties. The final developed platform shall be capable of autonomous Simultaneous Localisation and Mapping (SLAM), however, this will be the subject of a separate study.
\end{abstract}

Index Terms-Unmanned Aerial Vehicle, Nuclear Decommissioning, Nested Control, Trajectory Tracking, Sliding Mode Control.

\section{INTRODUCTION}

Unmanned Aerial Vehicles are becoming increasingly popular both in the consumer and commercial markets. Allowing for the ease of manoeuvrability, quadrotors provide an excellent basis to create a platform, based on which it is possible to survey and map unknown and hazardous environments for various purposes. This study builds on the previous work carried out in the Engineering Department at Lancaster University on the use of robotics and autonomous systems for nuclear decommissioning applications [1], [2]. Although the hydraulically actuated robotic manipulators play an important role for decommissioning tasks, they introduce significant challenges for modelling and control due to the inherent nonlinearities in the hydraulic joints. For example, in [3] a genetic algorithm technique is proposed to estimate the unknown parameters of a seven degree of freedom, hydraulically actuated manipulator in an output error setting. The estimation results are improved in [4] by converting the output error system identification cost

This work has been supported by the Centre for Innovative Nuclear Decommissioning (CINDe), which is led by the National Nuclear Laboratory, in partnership with Sellafield Ltd. and a network of Universities that includes the University of Manchester, Lancaster University, the University of Liverpool and the University of Cumbria. The authors would also like to acknowledge the Engineering and Physical Sciences Research Council (EPSRC), grant number EP/R02572X/1, and National Centre for Nuclear Robotics (NCNR). function to a multi-objective cost function using the multiobjectivisation technique. Further to the unknown parameters of the robot, its hyper redundant nature necessitates a novel and numerically efficient inverse kinematic algorithm to be robust against possible singularities [5]. The preliminary investigations on the methods to capture the nonlinearities of the hydraulic manipulator are explained in [6], [7]. The aim is to use these results for state-dependent control of the robot joints in the presence of Wiener type nonlinearity [8], [9]. An effective nuclear decommission solution requires a sufficient amount of time for pre-planning to collect and process the necessary information without human intervention. Having an autonomous unmanned aerial vehicle working with the manipulator can bring significant advantage to speed up and improve the accuracy and success of the decommissioning solution [10]. A quadrotor would allow faster 3D mapping and entrance into areas which may require copious amounts of planning and manpower to gain access to.

In [11], [12] a novel multi-channel chattering free robust nonlinear control system is designed for attitude control of quadrotor, in the presence of uncertainties and the direct wind disturbance on the model of the system. Recent developments to get closer to the practical implementation of the controller includes applying the Extended Kalman Filter (EKF) for state estimation [13], and the use of an event-triggered particle filter to improve the energy management of the quadrotor in the state estimation procedure [14]. Although the achieved results are promising, they have not been tested on a real quadrotor platform. This requires the implementation of an additional on-board computer (OBC) to process the sensory data in realtime, for the simultaneous localisation and mapping of the quadrotor whilst it is in flight. The processed data can be sent to the ground control station (GCS) where the generated map will be analysed. Various techniques, including object detection, can be utilised to maximise the potential uses of this technology. Therefore, in this paper, we address the extra work needed to prepare a testbed for implementation and evaluation of the reviewed algorithms in an autonomous flying vehicle with the possibility of mapping the surrounding environments and navigating through the environment by avoiding obstacles. Following this introduction, the paper is organised by explaining the embedded hardware, electronics, as well as the body 
frame and mechanical design in section II. The dynamical model of a quadrotor is displayed in section III. The approach adopted to design the $6 \mathrm{DOF}$ flight controller is presented in section IV. The simulation results are discussed in section V. Finally, the paper is wrapped up by drawing some conclusions in section VI.

\section{EMBEDDED HARDWARE AND ElECTRONICS}

The basic platform used to develop the UAV as a complex mechatronic system relies on the Holybro S500 chassis. Using this initial frame we can first develop the software and algorithms along with the other hardware interfaces, before designing and implementing a new larger frame. The benefit of using this chassis was that the power distribution board was enclosed within the actual frame. This would remove the potential issues which may occur due to any short circuits. Moreover, this saves further space on the quadrotor which would be taken up by a physical power distribution board and opens up possibilities for the use of more peripherals.

The Motors and Rotor blades to be used are a 2212920 $\mathrm{KV}$ motor set and $9.4 \times 4.3$ rotor blades [15]. This set has the potential to provide a maximum thrust of $1200 \mathrm{~g}$ per motor. With an estimated overall weight of the quadrotor being $2 \mathrm{Kg}$, this provides an ample amount of thrust to ensure that the quadrotor will be stable and have enough power to remain in flight for a sufficient amount of time.

The embedded flight controller unit (FCU) for this project is chosen to be the Pixhawk 4 mini from Holybro. This FCU controls the quadrotor motors and houses various sensors which are paramount for controlling of the quadrotor. The Pixhawk 4 Mini is the smaller version of the Pixhawk 4, with significant benefit for this project, as it allows for the use of extra space as well as weight saving which increases the operational flight time of the system. The FCU will have the elementary flight control algorithm, uploaded into the system, which increases the robustness and level of the control that the UAV is capable of having. Within the Pixhawk is the inertial measurement unit (IMU) which senses both the linear and rotational acceleration of the quadrotor in conjunction with the gyroscopes inside to understand the quadrotor's orientation mid-flight. In addition to this, the Pixhawk also houses a magnetometer and barometer. These two sensors aid in sensing the orientation as a compass and the pressure of the air to calculate the relative height of the quadrotor. All of these onboard sensors are involved and utilised as the sensory feedback for the control as well as the navigation systems. A PX4 flow sensor board has also been added to the design which allows for tracking of the movements through an optical flow camera as well as implementing another height sensor with a sonar sensor. This will increase the reliability and stability of the system in various manoeuvres such as take-off and landing, and especially when the quadrotor will need to maintain a steady hold in its position during the hovering mode.

The quadrotor will also hold an OBC which in this case is the Nvidia Jetson Nano. The Jetson Nano will run the Robot Operating System (ROS), on top of which the SLAM and navigation algorithm will be installed. Furthermore, a 2D LiDAR and an Intel RealSense camera sensor are added to the platform for a better perception of the surrounding environment. The data collected by these sensors are sent to the Nvidia Jetson Nano for further process. This information, along with a SLAM algorithm, will allow the UAV to understand its current location with respect to the map and make sense of the surrounding indoor environment. This data is also sent to the ground control computer where it can be reviewed and analysed by the specialists who are using UAV for a specific application. The data is sent from the Pixhawk to the Jetson and down to the ground control computer using the MAVROS protocol, which enables the possibility to send ROS topics from each device allowing for easy transmission of data.

Numerous drone frame configurations exist that have a direct impact on the flight dynamics of the drone. Having this in mind, a quadrotor ( $\mathrm{x}$-frame) configuration was chosen as it allows for intuitive control [16].

The design of the drone itself was chosen to be modular to give more flexibility on installation of different components such as the arms and to allow replacement of any parts damaged after the flight test. To hold all components such as the sensors, battery, flight controller and the on-board computer, the drone is designed with three levels. Two of them to be large $22 \times 22 \mathrm{~cm}$ layers and the third a smaller $11 \times 11 \mathrm{~cm}$ layer. The space available on the drone is more than required, however, the available room enables future upgrade of the drone by adding more sensors and components to improve the functionality of the platform. Additionally, to ensure the safe operation of the drone during the flight and possible crashes a propeller guard and component protection is designed and attached to the base platform.

Currently, to test the implementation of navigation, perception and control, the s500 quadrotor frame was used [17]. Figure 1 shows this drone frame including the 2 layers that are used to hold the components. The battery is attached below the bottom layer.
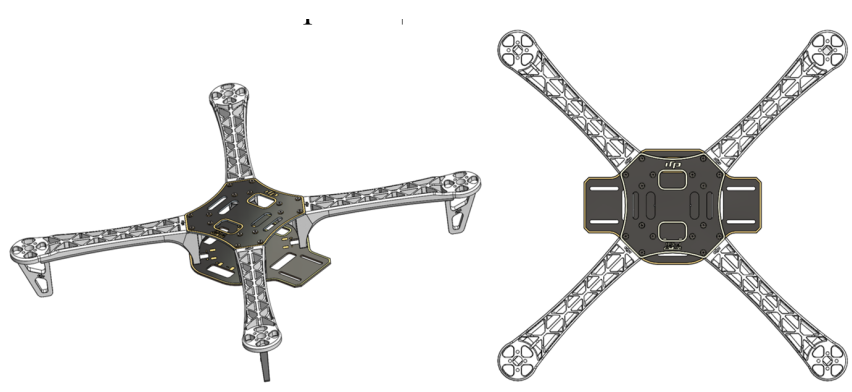

Fig. 1: S500 quadrotor frame from two angles.

\section{QUADROTOR MODEL}

The design of a control system for the quadrotor first requires the derivation of the mathematical model of the quadrotor. A diagram of an $\mathrm{x}$-frame quadrotor, with the rotor directions labelled, is shown in Figure 2. 


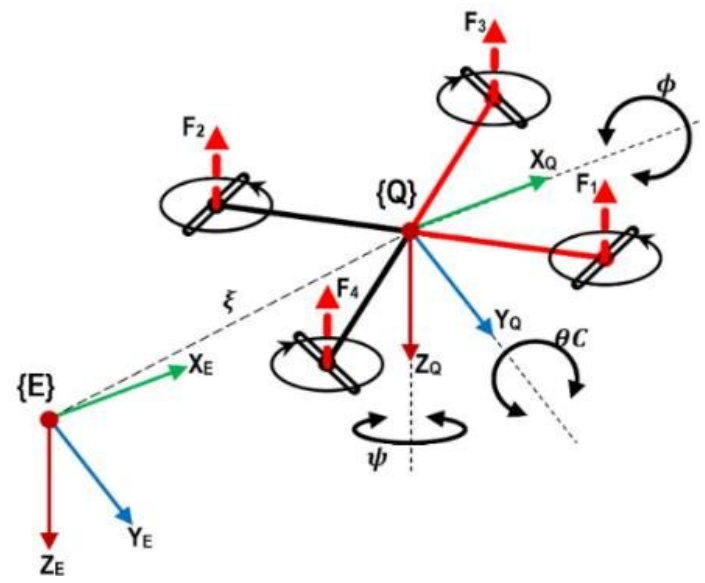

Fig. 2: Cross frame and the assigned coordinate systems [18].

$$
\omega=\left[\begin{array}{l}
\omega_{1} \\
\omega_{2} \\
\omega_{3} \\
\omega_{4}
\end{array}\right]
$$

where $\omega_{i}$ represents the angular velocity of the propeller $i$.

\section{A. Coordinate Frame}

First, the coordinate frame is defined for the position $(r)$ and attitude $(\alpha)$ of the quadrotor.

$$
r=\left[\begin{array}{l}
x \\
y \\
z
\end{array}\right], \alpha=\left[\begin{array}{l}
\varphi \\
\theta \\
\psi
\end{array}\right]
$$

Here, $\varphi, \theta$, and and $\psi$ are referring to the roll, pitch, and yaw angles and the vector $r$ determines the location of the quadcopter in 3D space. The Euler angles of the quadrotor can be used to determine how the rigid body of the quadrotor is oriented in space with a rotational matrix. The rotational matrix $(R)$ of the quadrotor is shown in (3).

$$
R=\left[\begin{array}{ccc}
c \varphi c \psi & c \psi s \varphi s \theta-c \varphi s \psi & c \varphi c \psi s \theta-s \varphi s \psi \\
c \varphi s \psi & s \varphi s \theta s \psi+c \varphi c \psi & c \varphi s \theta s \psi-c \psi s \psi \\
-s \theta & c \theta s \varphi & c \varphi c \theta
\end{array}\right]
$$

where $c \varphi=\cos (\varphi)$ and $s \varphi=\sin (\varphi)$.

\section{B. Linear Motion}

Using Euler's first law of motion for rigid body dynamics, the equations of linear motion for a quadrotor can be defined, where $K_{T}$ is the coefficient of thrust for the propellers and $m$ is the mass of the quadrotor.

$$
\begin{aligned}
& \frac{d^{2} r}{d t^{2}}=g\left[\begin{array}{l}
0 \\
0 \\
1
\end{array}\right]-\frac{R K_{T}}{m} \sum_{i=1}^{4} \omega_{i}^{2}\left[\begin{array}{l}
0 \\
0 \\
1
\end{array}\right] \\
& \ddot{x}=(-c \varphi c \psi s \theta-s \varphi s \psi) \frac{K_{T}}{m} \sum_{i=1}^{4} \omega_{i}^{2} \\
& \ddot{y}=(-c \varphi s \theta s \psi-c \psi s \varphi) \frac{K_{T}}{m} \sum_{i=1}^{4} \omega_{i}^{2}
\end{aligned}
$$

$$
\ddot{z}=g-c \varphi c \theta \frac{K_{T}}{m} \sum_{i=1}^{4} \omega_{i}^{2}
$$

\section{Rotational Motion}

The torques around each axis of the quadrotor are defined in equations (8)-(10) where $K_{T}$ is the coefficient of thrust of the propellers, $K_{D}$ is the coefficient of drag of the propellers, and $l$ is the length of each of the UAVs arms.

$$
\begin{aligned}
& \tau_{\varphi}=K_{T} l\left(\omega_{1}^{2}-\omega_{2}^{2}-\omega_{3}^{2}+\omega_{4}^{2}\right) \\
& \tau_{\theta}=K_{T} l\left(\omega_{1}^{2}+\omega_{2}^{2}-\omega_{3}^{2}-\omega_{4}^{2}\right) \\
& \tau_{\psi}=K_{D}\left(\omega_{1}^{2}-\omega_{2}^{2}+\omega_{3}^{2}-\omega_{4}^{2}\right)
\end{aligned}
$$

From Euler's second law of motion for rigid body dynamics, we get (11).

$$
\dot{\omega}_{\alpha}=I^{-1}\left(\tau_{\alpha}-\left(\omega_{\alpha} \times I \omega_{\alpha}\right)\right)
$$

where:

$$
\omega_{\alpha}=\left[\begin{array}{c}
\omega_{\varphi} \\
\omega_{\theta} \\
\omega_{\psi}
\end{array}\right]=\left[\begin{array}{c}
\dot{\varphi} \\
\dot{\theta} \\
\dot{\psi}
\end{array}\right], \tau_{\alpha}=\left[\begin{array}{c}
\tau_{\varphi} \\
\tau_{\theta} \\
\tau_{\psi}
\end{array}\right], I=\left[\begin{array}{ccc}
I_{x x} & 0 & 0 \\
0 & I_{y y} & 0 \\
0 & 0 & I_{z z}
\end{array}\right]
$$

From this, the equations of rotational motion can be derived.

$$
\begin{aligned}
& \frac{d \omega_{\varphi}}{d t}=\ddot{\varphi}=\frac{\left(I_{y y}-I_{z z}\right) \times \dot{\theta} \times \dot{\psi}}{I_{x x}}+\frac{\tau_{\varphi}}{I_{x x}} \\
& \frac{d \omega_{\theta}}{d t}=\ddot{\theta}=\frac{\left(I_{z z}-I_{x x}\right) \times \dot{\varphi} \times \dot{\psi}}{I_{y y}}+\frac{\tau_{\theta}}{I_{y y}} \\
& \frac{d \omega_{\psi}}{d t}=\ddot{\psi}=\frac{\left(I_{x x}-I_{y y}\right) \times \dot{\varphi} \times \dot{\theta}}{I_{z z}}+\frac{\tau_{\psi}}{I_{z z}}
\end{aligned}
$$

\section{System Inputs}

The inputs of the system are defined with the following matrix, where $u_{1}$ is the total thrust of the UAV and $u_{2}, u_{3}$, and $u_{4}$ are the rotational torques around the roll, pitch, and yaw axis respectively.

$U=\left[\begin{array}{l}u_{1} \\ u_{2} \\ u_{3} \\ u_{4}\end{array}\right]=\left[\begin{array}{l}u_{1} \\ \tau_{\varphi} \\ \tau_{\theta} \\ \tau_{\psi}\end{array}\right]=\left[\begin{array}{cccc}K_{T} & K_{T} & K_{T} & K_{T} \\ l K_{T} & -l K_{T} & -l K_{T} & l K_{T} \\ l K_{T} & l K_{T} & -l K_{T} & -l K_{T} \\ K_{D} & -K_{D} & K_{D} & K_{D}\end{array}\right]\left[\begin{array}{c}\omega_{1}^{2} \\ \omega_{2}^{2} \\ \omega_{3}^{2} \\ \omega_{4}^{2}\end{array}\right]$

Using these system inputs, the final linear and rotational equations of motion can be defined for the quadrotor.

$$
\begin{gathered}
u_{1}=K_{T} \sum_{i=1}^{4} \omega_{i}^{2} \\
\ddot{x}=(-c \varphi c \psi s \theta-s \varphi s \psi) \frac{u_{1}}{m} \\
\ddot{y}=(-c \varphi s \theta s \psi-c \psi s \varphi) \frac{u_{1}}{m} \\
\ddot{z}=g-c \varphi c \theta \frac{u_{1}}{m} \\
\ddot{\varphi}=\frac{\left(I_{y y}-I_{z z}\right) \times \dot{\theta} \times \dot{\psi}}{I_{x x}}+\frac{u_{2}}{I_{x x}}
\end{gathered}
$$




$$
\begin{aligned}
& \ddot{\theta}=\frac{\left(I_{z z}-I_{x x}\right) \times \dot{\varphi} \times \dot{\psi}}{I_{y y}}+\frac{u_{3}}{I_{y y}} \\
& \ddot{\psi}=\frac{\left(I_{x x}-I_{y y}\right) \times \dot{\varphi} \times \dot{\theta}}{I_{z z}}+\frac{u_{4}}{I_{z z}}
\end{aligned}
$$

\section{Flight Control System}

Sliding mode control is a robust nonlinear variable structure control method with effective disturbance rejection and robustness to parameter uncertainties. Disturbance rejection is particularly useful for implementation onto an autonomous UAV in real scenarios, where large amount of uncertainties resulting from corrosion and payload changes occurs for different missions. The situation gets worse due to changes in the environmental conditions such as wind, humidity, and radiation. Since the quadrotor dynamics belong to a class of unstable under-actuated dynamic systems, design and development of a six degree of freedom controller is not a straightforward task. A new chattering free sliding mode control method (CFSMC) for stabilisation and attitude control of UAV is proposed in [11].

A six degree of freedom sliding mode controller for the UAV is designed in the nested control form such that the position $(X Y)$, altitude $(Z)$, and attitude (roll, pitch and yaw) are separated into two distinct controllers. The altitude controller produces the desired overall thrust values, which are then used for position control. The position controller then outputs the desired roll and pitch values, leading directly into the attitude controller. Lastly, the desired and measured attitude values are manipulated to produce the thrust commands for each motor. The control laws for each of the three separate subsystems are laid out in the following.

\section{A. Chattering Free Sliding Mode Control}

First, the sliding surface is defined.

$$
\begin{aligned}
\sigma_{s} & =\dot{\widetilde{s}}+\lambda \widetilde{s} \\
\widetilde{s} & =s-s_{d}
\end{aligned}
$$

While classical sliding mode control presents a robust strategy for control, implementation often faces the issue of chattering. To alleviate the effect of chattering in design of the sliding mode control system we follow the method proposed in [11] by defining the Lyapunov function below

$$
V=\eta \times \sigma^{\frac{m}{n}} \times \operatorname{sign}(\sigma)
$$

To assure the stability of the system, the derivative of the Lyapunov candidate function must be negative. Therefore

$$
\frac{d V}{d t}=-\mu \times \sigma^{b} \times \operatorname{sign}(\sigma)<0 .
$$

Here $b$ is a fractional number between 0 and 1 with odd integers in numerator and denominator. By finding the time derivative of the Lyapunov candidate function (26), and inserting it into (27) we find

$$
\dot{\sigma_{s}}=-\frac{\mu}{\eta(m / n)} \times \sigma_{s}^{\left(b-\frac{m}{n}+1\right)}
$$

Finally, by substituting in the selected sliding surface we find

$$
\dot{\sigma_{s}}=\ddot{\widetilde{s}}+\lambda_{s} \dot{\widetilde{s}}=-\frac{\mu}{\eta(m / n)} \times \sigma_{s}^{\left(b-\frac{m}{n}+1\right)}
$$

By choosing $b$ such that $b<m / n$, it is easy to verify that $b-m / n+1$ can be written again as $\alpha / \beta$ with $\alpha$ and $\beta$ as positive odd integers and $\alpha<\beta$. Then (29) can be rewritten as

$$
\dot{\sigma_{s}}=\ddot{\widetilde{s}}+\lambda_{s} \dot{\widetilde{s}}=-\frac{\mu}{\eta} \times \sigma_{s}^{\left(\frac{\alpha}{\beta}\right)}
$$

\section{B. Altitude Control}

To control the $Z$ position of the quadrotor we start from the altitude dynamic in (20). By following the chattering free sliding mode control technique, derived in the previous section, the sliding surface for the altitude control $\sigma_{z}$ is given in (31). After some fairly standard mathematical manipulation, the total thrust $u_{1}$, applied to the drone can be calculated by knowing the measured and desired altitude values and their derivatives.

$$
\begin{gathered}
\sigma_{z}=\left(\dot{z}-\dot{z}_{d}\right)+\lambda_{z}\left(z-z_{d}\right) \\
u_{1}=\frac{m}{c \varphi c \theta}\left(\left(-\frac{\mu_{z}}{\eta_{z}} \times \sigma_{z}^{\left(\frac{\alpha z}{\beta z}\right)}\right)-\lambda_{z}\left(\dot{z}-\dot{z}_{d}\right)+g+\ddot{z}_{d}\right)
\end{gathered}
$$

In (32), $z$ and $z_{d}$ are the measured and desired $Z$ position, $\lambda_{z}$ as an undefined gain, and $g$ and $m$ are referring to gravity and mass respectively.

\section{Position Control}

By following a method similar to the one presented for the altitude control, it is possible to derive the robust control laws for $x$ and $y$ positions in (33) and (34). Here the desired roll $\varphi$ and pitch $\theta$ values are determined, based on the measured and desired $X$ and $Y$ positions.

$$
\begin{gathered}
u_{x}=\frac{m}{u_{1}}\left(\left(-\frac{\mu_{x}}{\eta_{x}} \times \sigma_{x}^{\left(\frac{\alpha_{x}}{\beta_{x}}\right)}\right)-\lambda_{x}\left(\dot{x}-\dot{x}_{d}\right)+g+\ddot{x}_{d}\right) \\
u_{y}=\frac{m}{u_{1}}\left(\left(-\frac{\mu_{y}}{\eta_{y}} \times \sigma_{y}^{\left(\frac{\alpha_{y}}{\beta_{y}}\right)}\right)-\lambda_{y}\left(\dot{y}-\dot{y}_{d}\right)+g+\ddot{y}_{d}\right) \\
\varphi_{d}=\arcsin \left(u_{x} \sin \left(\psi_{d}\right)-u_{y} \cos \left(\psi_{d}\right)\right) \\
\theta_{d}=\arcsin \left(\frac{u_{x} \cos \left(\psi_{d}\right)+u_{y} \sin \left(\psi_{d}\right)}{\cos \left(\varphi_{d}\right)}\right)
\end{gathered}
$$

\section{Attitude Control}

Leading from the position control, the attitude controller uses the desired attitude $\left[\begin{array}{lll}\varphi_{d} & \theta_{d} & \psi_{d}\end{array}\right]^{T}$ values, the moment of inertia of the quadrotor $I_{x x}, I_{y y}, I_{z z}$, and the current measured attitude $\left[\begin{array}{lll}\varphi & \theta & \psi\end{array}\right]^{T}$, to determine the the control commands $u_{2}, u_{3}$, and $u_{4}$.

$$
\begin{array}{r}
u_{2}=I_{x x}\left(\left(-\frac{\mu_{\varphi}}{\eta_{\varphi}} \times \sigma_{\varphi}^{\left(\frac{\alpha_{\varphi}}{\beta \varphi}\right)}\right)-\lambda_{\varphi}\left(\dot{\varphi}-\dot{\varphi}_{d}\right)+\ddot{\varphi}_{d}\right. \\
\left.-\left(\frac{I_{y y}-I_{z z} \times \dot{\theta} \times \dot{\psi}}{I_{x x}}\right)\right)
\end{array}
$$




$$
\begin{array}{r}
u_{3}=I_{y y}\left(\left(-\frac{\mu_{\theta}}{\eta_{\theta}} \times \sigma_{\theta}^{\left(\frac{\alpha_{\theta}}{\beta_{\theta}}\right)}\right)-\lambda_{\theta}\left(\dot{\theta}-\dot{\theta}_{d}\right)+\ddot{\theta}_{d}\right. \\
\left.-\left(\frac{I_{z z}-I_{x x} \times \dot{\varphi} \times \dot{\psi}}{I_{y y}}\right)\right) \\
u_{4}=I_{z z}\left(\left(-\frac{\mu_{\psi}}{\eta_{\psi}} \times \sigma_{\psi}^{\left(\frac{\alpha_{\psi}}{\beta_{\psi}}\right)}\right)-\lambda_{\psi}\left(\dot{\psi}-\dot{\psi}_{d}\right)+\ddot{\psi}_{d}\right. \\
\left.-\left(\frac{I_{x x}-I_{y y} \times \dot{\varphi} \times \dot{\theta}}{I_{z z}}\right)\right)
\end{array}
$$

Equations (37), (38), and (39) also use the same notation as (32)-(34). By putting the four thrust commands in the vector $U$ it is possible to derive the motor speeds using (16). As such, the CFSMC controller is designed in MATLAB.

\section{Simulation}

To evaluate the effectiveness of the different control systems, simulations were carried out in MATLAB using the dynamical model of the quadrotor. The results from the CFSMC were compared with classical sliding mode control and PID control systems. A change in the payload is introduced to evaluate the robustness of different control approaches.

The simulations implement a nonlinear airframe as well as a sensor block to simulate sensor noise. This allows a more realistic comparison of the control systems.

\section{A. No Disturbance}

First, we evaluate the performance of different control systems for a hovering scenario with no disturbance input. The controllers are evaluated by taking the Integral Absolute Error (IAE) of the quadrotor position in $x, y$, and $z$ directions. The results are summarised in Table I.

TABLE I: IAE for Quadrotor Positions with No Disturbance

\begin{tabular}{|c|c|c|c|}
\hline Integral of & \multicolumn{3}{|c|}{ Control Systems } \\
\cline { 2 - 4 } Absolute Error & $\boldsymbol{P I D}$ & SMC & CFSMC \\
\hline X Position & 0.3154 & 0.4368 & 0.2090 \\
\hline Y Position & 0.8131 & 0.3519 & 0.2341 \\
\hline Z Position & 4.9920 & 4.5440 & 4.4320 \\
\hline
\end{tabular}

The transient response of the quadrotor can be evaluated by moving the quadrotor through step responses along each axis in a sequence. It is assumed that the quadrotor is initially positioned at $(x, y, z)=(0,0,0)$. The flight scenario includes a step change to the desired position $\left(x_{d}, y_{d}, z_{d}\right)=(0,0,1)$ at $t=0$, then it is moving to $\left(x_{d}, y_{d}, z_{d}\right)=(0,1,1)$ at $t=10$, and when $t=20,\left(x_{d}, y_{d}, z_{d}\right)=(1,1,1)$. The simulation results are depicted in Figure 3.

\section{B. Payload Disturbance}

To evaluate the robustness of different controllers, they are compared by changing the payload as a disturbance input during the hovering. The mass, and inertial values of the quadrotor were each increased by $25 \%$ and the controllers
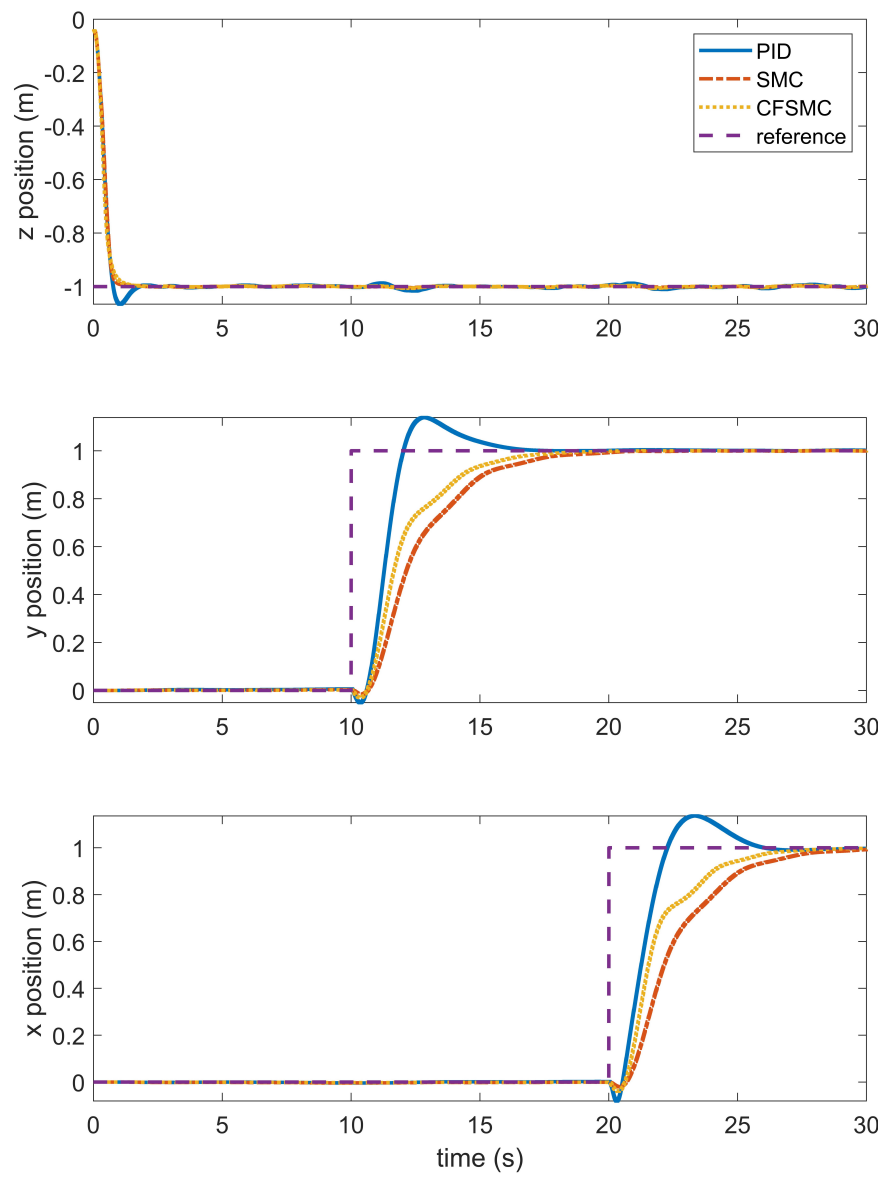

Fig. 3: Transient response of three control techniques with a sequence of step inputs

TABLE II: IAE for Quadrotor Positions with 25\% Payload Increase

\begin{tabular}{|c|c|c|c|}
\hline \multirow{2}{*}{ Integral of } & \multicolumn{3}{|c|}{ Control Systems } \\
\cline { 2 - 4 } Absolute Error & $\boldsymbol{P I D}$ & $\boldsymbol{S M C}$ & $\boldsymbol{C F S M C}$ \\
\hline X Position & 0.6650 & 0.4851 & 0.2330 \\
\hline Y Position & 0.9531 & 0.4661 & 0.2542 \\
\hline Z Position & 60.5954 & 9.2040 & 6.4362 \\
\hline
\end{tabular}

were compared again. Table II shows the of the IAE of quadrotor position in $x, y$, and $z$ directions.

The system was then evaluated with the sequence of step inputs with the $25 \%$ payload increase. The results show that the chattering free sliding mode technique shows improved performance over classical sliding mode, while both systems are more robust than PID for position control of a quadrotor in the presence of disturbance.

\section{CONCLUSions}

Overall, within the scope of the project, a complex mechatronic system has been designed and is under development to maintain a robust embedded platform which is capable of autonomous navigation in an unknown environment through mapping of the surrounding environment and localising itself 

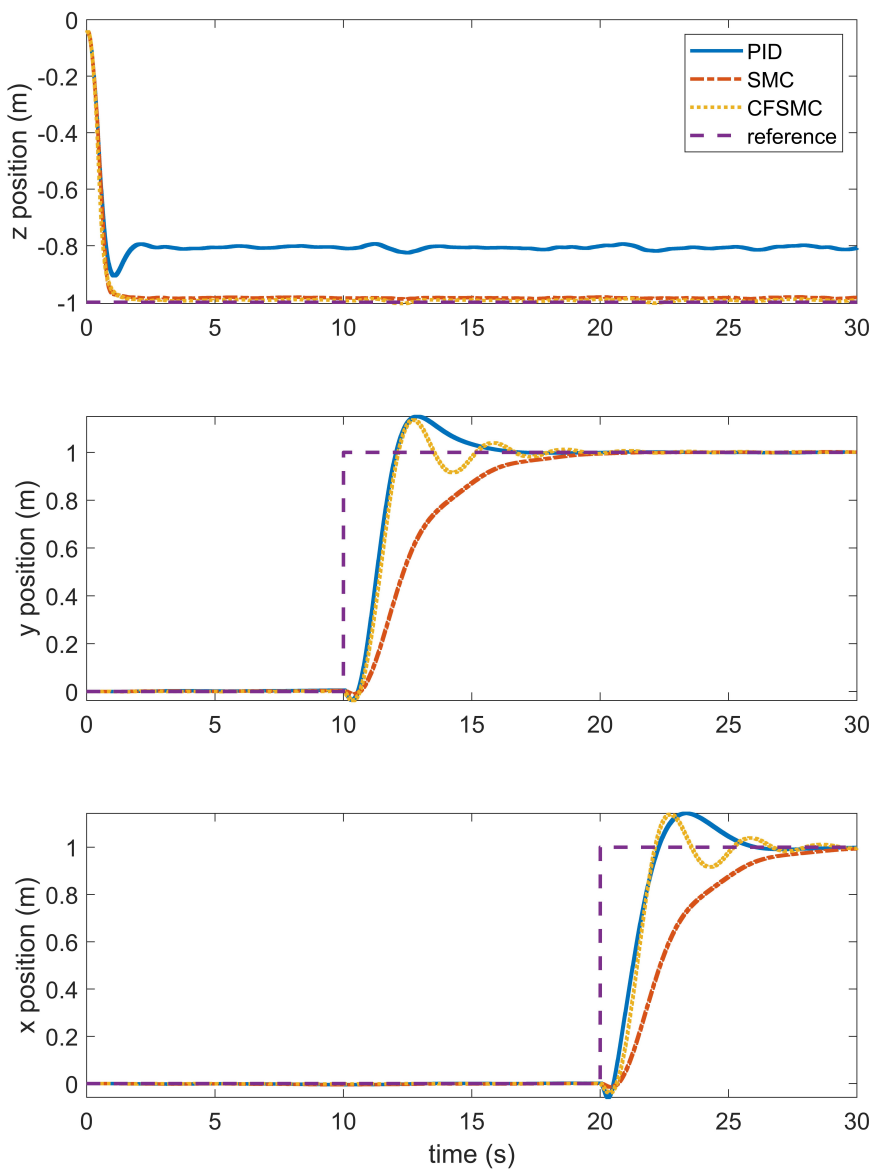

Fig. 4: Transient response of three control techniques with a sequence of step inputs and $25 \%$ increase in payload

within the map. An advanced control system, based on sliding mode control, has been developed and tested in simulation and shows promising results over PID and classical SMC for use in situations with disturbances. The main prospective application of this type of this mechatronic system is in nuclear decommissioning, where humans cannot enter easily due to the inaccessibility and hazardous nature of the facilities. It can be also used to enhance the overall situational awareness of the manipulators used for decommissioning to aid in decommissioning tasks. Future work involves implementing and testing the control system on the designed quadrotor to evaluate it experimentally, as well as integrating all components discussed in this paper to create a working prototype of the mechatronic system.

\section{REFERENCES}

[1] A. Montazeri and J. Ekotuyo, "Development of dynamic model of a 7DOF hydraulically actuated tele-operated robot for decommissioning applications," in Proceedings of the American Control Conference, vol. 2016-July. Institute of Electrical and Electronics Engineers Inc., jul 2016, pp. 1209-1214.

[2] A. Montazeri, C. West, S. D. Monk, and C. J. Taylor, "Dynamic modelling and parameter estimation of a hydraulic robot manipulator using a multi-objective genetic algorithm," International Journal of Control, vol. 90, no. 4, pp. 661-683, apr 2017.
[3] C. West, A. Montazeri, S. D. Monk, and C. J. Taylor, "A genetic algorithm approach for parameter optimization of a 7DOF robotic manipulator," IFAC-PapersOnLine, vol. 49, no. 12, pp. 1261-1266, 2016.

[4] C. West, A. Montazeri, S. D. Monk, D. Duda, and C. J. Taylor, "A new approach to improve the parameter estimation accuracy in robotic manipulators using a multi-objective output error identification technique," in RO-MAN 2017 - 26th IEEE International Symposium on Robot and Human Interactive Communication, vol. 2017-January. Institute of Electrical and Electronics Engineers Inc., dec 2017, pp. 1406-1411.

[5] T. Burrell, A. Montazeri, S. Monk, and C. J. Taylor, "Feedback Control-Based Inverse Kinematics Solvers for a Nuclear Decommissioning Robot," IFAC-PapersOnLine, vol. 49, no. 21, pp. 177-184, 2016.

[6] A. Oveisi, A. Anderson, T. Nestorović, and A. Montazeri, "Optimal Input Excitation Design for Nonparametric Uncertainty Quantification of Multi-Input Multi-Output Systems," IFAC-PapersOnLine, vol. 51, no. 15 , pp. 114-119, jan 2018.

[7] A. Oveisi, T. Nestorović, and A. Montazeri, "Frequency Domain Subspace Identification of Multivariable Dynamical Systems for Robust Control Design," IFAC-PapersOnLine, vol. 51, no. 15, pp. 990-995, jan 2018.

[8] C. West, S. D. Monk, A. Montazeri, and C. J. Taylor, “A Vision-Based Positioning System with Inverse Dead-Zone Control for Dual-Hydraulic Manipulators," in 2018 UKACC 12th International Conference on Control, CONTROL 2018. Institute of Electrical and Electronics Engineers Inc., oct 2018, pp. 379-384.

[9] C. West, E. D. Wilson, Q. Clairon, S. Monk, A. Montazeri, and C. J. Taylor, "State-dependent parameter model identification for inverse dead-zone control of a hydraulic manipulator*," IFAC-PapersOnLine, vol. 51, no. 15, pp. 126-131, jan 2018.

[10] T. Burrell, C. West, S. D. Monk, A. Montezeri, and C. J. Taylor, "Towards a Cooperative Robotic System for Autonomous Pipe Cutting in Nuclear Decommissioning," in 2018 UKACC 12th International Conference on Control, CONTROL 2018. Institute of Electrical and Electronics Engineers Inc., oct 2018, pp. 283-288.

[11] H. Nemati and A. Montazeri, "Design and Development of a Novel Controller for Robust Attitude Stabilisation of an Unmanned Air Vehicle for Nuclear Environments," in 2018 UKACC 12th International Conference on Control, CONTROL 2018. Institute of Electrical and Electronics Engineers Inc., oct 2018, pp. 373-378.

[12] _ "Analysis and Design of a Multi-Channel Time-Varying Sliding Mode Controller and its Application in Unmanned Aerial Vehicles," IFAC-PapersOnLine, vol. 51, no. 22, pp. 244-249, jan 2018.

[13] _ _ " "Output Feedback Sliding Mode Control of Quadcopter Using IMU Navigation," in Proceedings - 2019 IEEE International Conference on Mechatronics, ICM 2019. Institute of Electrical and Electronics Engineers Inc., may 2019, pp. 634-639.

[14] N. Sadeghzadeh Nokhodberiz, H. Nemati, and A. Montazeri, "EventTriggered Based State Estimation for Autonomous Operation of an Aerial Robotic Vehicle," Tech. Rep., 2019.

[15] "Holybro - Pixhawk4 mini." [Online]. Available: https://www.3dxr.co.uk/product/pixhawk4-mini/

[16] L. Camilli, "Emerging technologies, applications, regulations, and market challenges in the consumer aerial drone industry: A strategic analysis of the 3D Robotics Business Model," 2015.

[17] "Holybro - S500 V2 Frame Only Kit." [Online]. Available: https://www.3dxr.co.uk/product/holybro-s500-v2-frame-only-kit/

[18] A. Noordin, A. Basri, and Z. Mohamed, "Sensor fusion for attitude estimation and pid control of quadrotor uav," International Journal of Electrical and Electronic Engineering Telecommunications., vol. 7, pp. 183-189, 102018. 